\title{
Incidence of Mandibular Third Molar Impaction in Patients Visiting a Private Dental College
}

\author{
Sonti Sri Harsha, (CRRI) \\ Saveetha Dental College, Saveetha University, Chennai, India
}

\begin{abstract}
The study aims to evaluate the incidence of types of impaction of mandibular third molars. Since mandibular third molars are the most common teeth to get impacted, it is important to know the incidence and prevalence in a particular community. The study is done using radiographic records of patients visiting the department of oral and maxillofacial surgery, with a diagnosis of an impacted third molar that has the need to undergo extraction. The sample size was of 100 patients, all above 16 years of age. The orthopantomogram and intra oral periapical radiographs of the patients were interpreted to analyze the pattern of impacted mandibular third molars. The type of impaction was determined using Winter's Classification of Impacted Teeth (1926). Based on these values the incidence of type of impaction was determined.
\end{abstract}

Keywords: Impaction, Incidence, Mandibular third molars.

\section{Introduction}

Third molars are the most common teeth that are impacted among all molars ${ }^{1}$. The reason for their extraction could be due to its impaction or its potential complications damaging the adjacent teeth or the involved tooth itself. Impaction is cessation of eruption of a tooth caused by a physical barrier or ectopic positioning of a tooth ${ }^{2}$. An impacted tooth is one that is erupted, partially erupted or unerupted and will not eventually assume a normal arch relationship with other teeth and tissues ${ }^{2}$.

Impactions can be classified based on the position of the impacted third molar with the long axis of the second molars. Some of the types include: Mesio-angular, disto-angular, horizontal, and vertical types. Of all these the most common would be the mesio-angular variety.

This study focusses on the evaluation of the incidence of type of impaction of the mandibular third molars.

\section{Materials And Methods}

This study was conducted in the Department of Oral and Maxillofacial Surgery, for a period of three months from June to August 2013, on patients who required surgical removal of the impacted tooth.

The sample size of the study was 100, all above 16 years of age.

All the patients were assessed either with the orthopantomogram or IOPA.

The inclusion criteria of the study group are as follows:

- OPGs and IOPAs of the patients requiring surgical removal of the impacted tooth.

- Patients with no history of trauma.

- Patients with no syndromes and/or no systemic disorders.

The radiographs were analyzed with a radiographic viewer and magnifying lenses. Interpretation of the various radiographs showed the following:

- Impacted mandibular third molars

- Angulation of impacted mandibular third molars

\section{Results}

In this study a total number of 100 patients with mandibular third molars being impacted were included on an age scale between 15 to 65 years, out of which 58 are males and 42 are females.

The type of impaction was diagnosed with the help of IOPA (Intra Oral Periapical Radiograph) and OPG (Orthopantomograph) records of each patient based on Winter's Classification (1926) ${ }^{3}$.

The incidence of mesio-angular type was 63, out of which 35 were from males and 28 from females.

The incidence of disto-angular variant was 10, out of which 5 were from males and 5 from females.

The incidence of horizontal variant was 26, out of which 17 were from males and 9 from females.

The incidence of vertical type was 1 which was from a male patient. 
In this study it was observed that mesio-angular variant was the most common type to occur. Results suggested that males were the commonly affected ones. It was also observed that 26-32 years of age was the most common which witnessed occurrence of this type of impaction with maximum number of patients exhibiting the feature. Age wise distribution was done accordingly -

1) $15-25$ years

2) 26-35 years

3) $36-45$ years

4) $46-55$ years

5) 56-65 years

On comparing these age groups, incidence of different types of impactions according to the age is as follows:

Table 1: Age wise distribution of different types of impaction Age groups

\begin{tabular}{|cl|c|c|c|c|c|}
\hline & Type of impaction & $15-25$ years & $26-35$ years & $36-45$ years & $46-55$ years & $56-65$ years \\
\hline 1) & Mesio-angular type & 19 & 32 & 9 & 2 & 1 \\
\hline 2) & Disto-angular type & 2 & 5 & 3 & 0 & 0 \\
\hline 3$)$ & Horizontal type & 8 & 13 & 4 & 0 & 0 \\
\hline 4$)$ & Vertical type & 1 & 0 & 0 & 1 & 0 \\
\hline
\end{tabular}

Percentage distribution of the incidence of lower mandibular impaction in patients visiting a private dental college is as follows.

1. The incidence of mesio-angular type in 100 patients was $63 \%$, out of which $35 \%$ was from males and $28 \%$ from females.

2. The incidence of disto-angular type in 100 patients was $10 \%$, out of which $5 \%$ was from males and $5 \%$ from females.

3. The incidence of horizontal type in 100 patients was $26 \%$, out of which $17 \%$ was from males and $9 \%$ from females.

4. The incidence of vertical type in 100 patients was $1 \%$. This was a male patient's inference.

From the above study, it can be concluded that

\section{Conclusion}

1. Mesioangular is the most common variant of impaction seen in both males and females.

2. The percentage of mesioangular variant is more common in males than in females.

3. Horizontal impaction is the second most common pattern and more common in males than in females.

4. Vertical impaction is the least observed pattern of impaction in both males and females.

\section{References}

[1] Ramamurthy A, Pradha J, Jeeva S, Jeddy N, Sunitha J, Kumar S. Prevalence of Mandibular Third Molar Impaction and Agenesis: A Radiographic South Indian Study. J Indian Aca Oral Med Radiol, 24(3), 2012, 173-176.

[2] Neelima Anil Malik, Textbook of Oral and Maxillofacial Surgery (Daryaganj, New Delhi :Jaypee 2008) $122-123$.

[3] S M Balaji, Textbook of Oral and Maxillofacial Surgery (Ajantha offset, New Delhi :Elsevier 2007) 233. 